\title{
An Evaluation Model for Selecting Cloud Services from Commercially Available Cloud Providers
}

\author{
Shyam S. Wagle*, Mateusz Guzek ${ }^{\dagger}$, Pascal Bouvry ${ }^{\dagger}$, Raymond Bisdorff ${ }^{\dagger}$ \\ University of Luxembourg \\ 6, rue R. Coudenhove-Kalergi, Luxembourg City, Luxembourg \\ * Email: shyam.wagle.001@student.uni.lu \\ † Email: firstname.lastname@uni.lu
}

\begin{abstract}
Selecting the appropriate cloud services and cloud providers according to the cloud users requirements is becoming a complex task, as the number of cloud providers increases. Cloud providers offer similar kinds of cloud services, but they are different in terms of price, quality of service, customer experience, and service delivery. The most challenging issue of the current cloud computing business is that cloud providers commit a certain Service Level Agreement (SLA), with cloud users, but there is little or no verification mechanisms which ensure that cloud providers are providing cloud services according to their commitment. In the current literature, there is a lack of an evaluation model which provides the real status of cloud providers for the cloud users. In this paper, an evaluation model is proposed, which verifies the quality of cloud services delivered for each service and provides the service status of the cloud providers. Finally, evaluation results obtained from cloud auditors are visualized in an ordered performance heat map, showing the cloud providers in a decreasing ordering of overall service quality. In this way, the proposed service quality evaluation model represents a visual recommender system for cloud service brokers and cloud users.
\end{abstract}

Index Terms-Cloud Computing; Service Measurement; Service Verification; Service Evaluation Model; Recommender System ; Provider Selection ; Performance Heat Map

\section{INTRODUCTION}

With the increasing number of Cloud Service Providers (CSPs), selecting the appropriate CSPs according to their requirements is a complex and tedious job for cloud users. During the service negotiation process, the cloud user and the cloud provider agree on the contract which includes the terms and conditions to be followed by both parties. This contract is called Service Level Agreement (SLA). SLAs are composed of different Quality of Service (QoS) rules, which are the obligations that have to be followed by the cloud providers [1]. A Cloud provider is a subject to contractual compensations in case of SLA breaches. This penalty is as essential to the SLA as guarantees, a notion essential to the very concept of using SLAs as instruments for providing some level of determinism in business relations [2]. Thus an SLA should also describe what shall happen when something goes wrong, that is when SLA is violated.

In the current practice, cloud providers offer SLA templates on their websites, and cloud users agree on the corresponding SLA terms and conditions while subscribing the cloud services. If a cloud provider does not meet the committed service offer, cloud users are eligible to receive a Service Credit. The service credit is a credit calculated as a percentage of the total charges paid by the cloud user. The received compensation may be however negligible compared to the losses caused by the insufficient QoS.

Due to the lack of the standardized SLA template in cloud computing, cloud providers are not legally obliged to offer any specific SLA template. Majority of the cloud providers offer monthly uptime (in percents) as the main QoS indicator. Other attributes are not clear in terms of SLA service and monitoring. In many cases, cloud providers offer similar or identical characteristics of services such as service availability, reliability, but they exhibit differences in terms of price, quality of service, service delivery and customer experience. This specific adds an extra burden on cloud users and cloud brokers when they select cloud services from cloud providers [3].

In this paper, a service evaluation model which measures cloud services delivered by commercially available cloud providers using cloud auditors is presented. It verifies the service delivery compliance with the committed SLA offer, however verification of services is limited to the measurable parameters accessible from the majority of cloud providers. Those values are further visualized in a performance tableau format showing the evaluated cloud service providers in a decreasing order of overall performance. The main contributions of the paper are:

1) Measurement of cloud services delivered by commercially available cloud providers.

2) Verification of cloud services delivered by cloud providers according to SLAs offered by cloud providers.

3) Evaluation of cloud providers on a hierarchy of performance criteria.

4) Multiple criteria ranking of cloud providers that aids cloud users and cloud brokers in selection of the appropriate cloud services.

The paper is organized as follows: Section II describes the related works in CSP ranking and service selection. Section III provides the framework for SLA assured brokering and recommendation model. The SLA attributes measured to evaluate the CSPs are briefed in section IV. The measured parameters and verification of cloud services with offered SLA are presented in section V. A novel CSP selection aid is 
proposed in VI. Service selection recommendation is discussed in section VII. Conclusions and future works directions are described in section VIII.

\section{RELATED WORKS}

In the current growing cloud computing business, selecting the best cloud service from appropriate cloud providers is very complex and challenging for the cloud users. There is a multitude of works that employ optimization to achieve this goal [4], however they usually require certain input data, and their solutions are applicable for the current situation. The multi-objective optimization attempts [5] overcome some of these limitations, but in turn they provision a Pareto front of optimal solutions, which creates a consecutive problem of the selection of the final solution .

The selection of the best web services according to consumers' opinion for the web service selection based on consumer's vague perception is introduced by Wang [6]. Practically, web services and cloud services can not be evaluated only on the basis of consumers' perception, because user may not receive actual service delivered by cloud provider due to network problem, e.g. limited bandwidth, or other problems.

For the ranking of cloud services SMICloud [7] has proposed a framework for comparing and ranking cloud services. It considers only quantifiable SLA attributes defined by Cloud Service Measurement Index Consortium (CSMIC) [8], and does not consider the qualitative attributes. SMICloud is based on an analytic hierarchy process (AHP) [9]. The main difficulty in the provider ranking based on the AHP technique is assigning the hierarchy of SLA attributes. In practice, each SLA attributes are important and dependent on each user's preferences.

The authors in CloudCmp [10] have proposed a framework to compare the performance of different Cloud services such as Amazon EC2, Windows Azure and Rackspace, but it only compares the low-level performance metrics of Cloud services such as CPU utilization and network throughput etc. Such low-level performance metrics could be further used to create models of high-level system properties, such as power consumption or performance [11], but it has not been tackled.

Hoi Chan et. al [12] have proposed ranking and mapping of cloud computing applications. It is limited to few SLA attributes and does not provide a basis on which the weights of cloud services are assigned to each cloud provider. Service Ranking System [13] searches SLA offers provided by cloud providers rather than the quality of delivered services to rank the cloud providers. The authors in CloudRank [14] propose a cloud ranking algorithm based on greedy method which considers few functional parameters to rank the cloud providers and does not consider the delivered services in their framework. The cloud service selection process based on consumer experience and involving the third party to avoid a biased assessment of cloud services from users has been proposed by Qu et. al [15], however it does not cover the performance measurements from cloud providers. To the best of our knowledge, there is no evaluation framework which

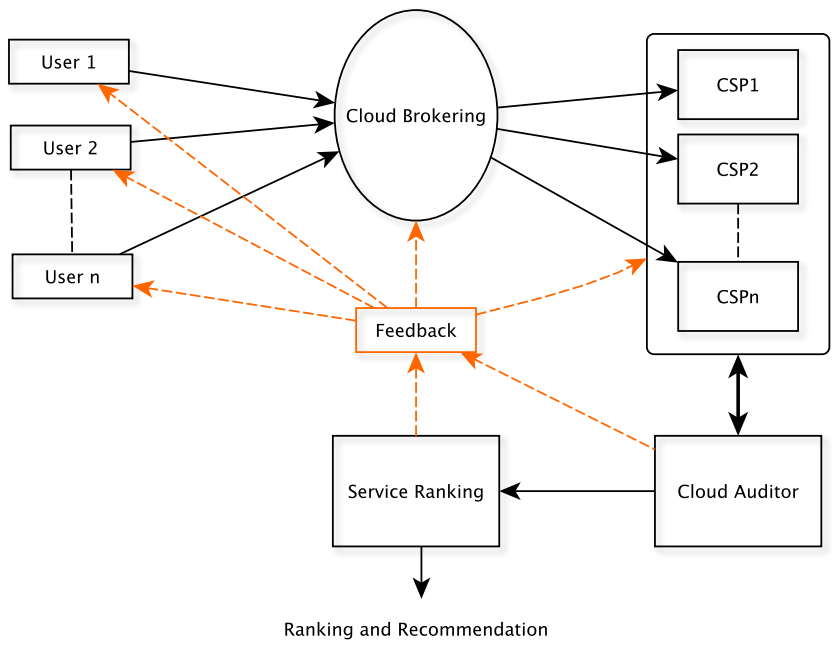

Fig. 1. Proposed Framework for SLA-based Service Brokering

selects and ranks the commercially available cloud providers based on their delivery of service compared with the SLAs offered by them.

\section{SLA BASED CLOUD SERVICE BROKERING FRAMEWORK}

Fig. 1 shows the overall framework for SLA assured brokering based on the service delivery of cloud providers and the service experience of cloud users. Cloud auditors measure the service performance delivered by cloud providers. The Cloud Auditor module contains cloud auditors (Auditor ${ }_{1}$ and Auditor $_{2}$ ). In our case both cloud auditors collects performance of commercially available cloud providers. The Cloud Auditor module also compares the service delivered by cloud providers with SLA commitment to cloud users. If a cloud provider is delivering services according to its commitment, the cloud provider is verified. Otherwise the cloud provider is not verified.

The Service Ranking system ranks and recommends the cloud providers based on their SLAs and effective service delivered by cloud providers. The service ranking and recommendation system provides overall performance ranking of cloud providers. Based on the information provided by the Service Ranking module, the Feedback module distributes information about each cloud provider to cloud broker and cloud users. To improve the quality of performance measurement and customer feedback, multiple cloud auditors and cloud users can be involved. Additionally, in case of information from one source, other sources still can provide the status of cloud providers' services. Different independent auditors also make the system more trustworthy. The cloud broker collects the requirements of cloud users and recommends cloud services from different cloud providers based on ranking and service performance provided by the feedback system. The SLAassured cloud brokering is however out of scope in this paper. 


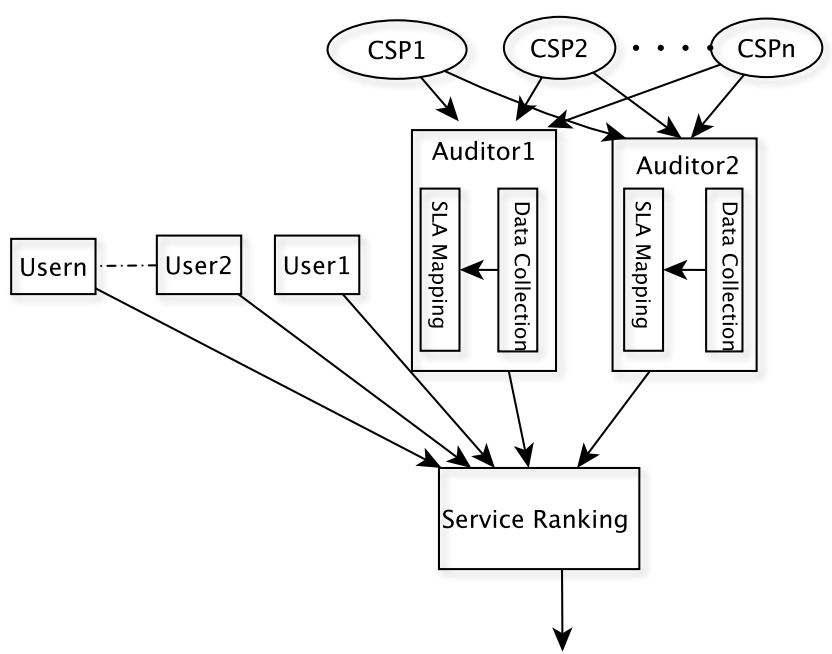

Service Evaluation and Recommendation

Fig. 2. Recommendation Model

Overview of our solution is shown in Fig. 2. Low-level data are collected from commercially available cloud providers. These values are mapped to SLA parameters so that the measured performance can be compared with SLA offer by cloud providers. The Cloud Auditor (module of service monitor and service verifier) verifies the services delivered by cloud providers. The service ranking module collects the service measurement and user experience from cloud auditors and cloud users respectively, to provide cloud provider ranking and service recommendation to the cloud users.

\section{Cloud COMPUTING SERVICE METRICS}

Cloud service metrics provide knowledge about characteristics of a cloud service by their definitions (e.g. expression, unit, rules) and the values resulting from the observation of the property [16]. It is necessary to define a set of specific metrics, on basis of which cloud services are selected. The metrics play an important role in the evaluation, as well as the selection of the cloud providers. In case of cloud computing, there are no standard, uniform SLA metrics and it is not mandatory for cloud providers to follow any specific metric. It is thus more challenging to consider all the important aspects of a standard cloud computing SLA.

Some initiative works have been carried out to define the SLA metrics in cloud computing. The selected in this study SLA metrics used to assess the cloud services from appropriate cloud providers comes from the literature. Guide to Cloud SLA [17], Service Measurement Index (SMI) defined by CSMIC, TM Forum [18], NIST Cloud Computing Standards Roadmap [16], Cloud Computing Service Level Agreements [19], OCCI working group [20] have provided their important contributions to standardize the SLA metrics in cloud computing.

In this work, five main performance criteria are chosen as important requirements to measure QoS for the cloud
TABLE I

CRITERIA AND SUB-CRITERIA FOR EVALUATING CLOUD SERVICES

\begin{tabular}{|l|l|l|}
\hline Criteria & Sub-criteria & Short Name \\
\hline Availability (C1) & Uptime(c11) & $u p T$ \\
& Downtime(c12) & $d w T$ \\
& Outage Frequency(c13) & ouT \\
\hline Reliability (C2) & Load Balancing(c21) & LB \\
& MTBF(c22) & $M T B F$ \\
& Recoverable(c23) & Rcv \\
\hline Performance (C3) & Latency(c31) & Lat \\
& Response time(c32) & $r s T$ \\
& Throughput (c33) & $t p T$ \\
\hline Cost (C4) & Storage Cost (c41) & $s t C$ \\
& VM instance cost(c42) & $s n C$ \\
\hline Security (C5) & Authentication(c51) & $a u T$ \\
& Encryption(c52) & enC \\
& Audit-ability(c53) & $a u D$ \\
\hline
\end{tabular}

users: Availability, Reliability, Performance, Cost and Security. Under each main criteria, subcritera, which are directly measurable from cloud provider premises, are defined. To cover measurable and non-measurable preferential aspects in the evaluation model, we have selected five SLA attributes; Performance, Availability and Reliability as functional criteria, Security and Cost as a non-functional criteria. To map measured sub-criteria to SLA attributes, we use mapping rules. For example, if service downtime and uptime of particular cloud provider are known then they are mapped into an Availability performance as follows:

$$
A_{v}=\frac{\text { uptime }}{\text { uptime }+ \text { downtime }}
$$

Mapping to SLA criteria from measured sub-criteria values depends on the nature of services and types of service used. Definitions [1] and mathematical formula provided in [21], [22], [23] are used in criteria mapping.

\section{Verification And Evaluation of Cloud PROVIDERS}

\section{A. Service Performance Measurement}

To evaluate the service performance of the eleven selected cloud providers (see Table II), two cloud auditors are used: CloudAuditor $_{1}$, CloudAuditor 2 . Evaluation data presented in Table III and Table IV was collected over a period of seven days. Internet connection between local test environment and the cloud providers is presented in Table II. CloudAuditor 1 uses CloudHarmony (www.cloudharmony.com) monitoring tool and CloudAuditor 2 uses monitis (www.monitis.com) monitoring tool. Service performance is measured for all cloud users based in Luxembourg. The verification of cloud services of a cloud provider with a committed SLA is difficult because cloud providers often do not provide sufficient information in their SLA and they are not legally obliged to provide all the information in their SLA. The selected cloud providers provide only monthly uptime of service delivery.

All services are measured on the basis of SLA attributes defined in Table I. Missing data are represented as ' $N A$ '. Measurements of cloud service performance of each cloud 
TABLE II

INTERNET CONNECTION BETWEEN LOCAL TEST ENVIRONMENT AND CLOUd PROVIDERS

\begin{tabular}{|l|l|l|l|l|l|}
\hline SN & Cloud Provider & Short Name & Website & Downlink (Mbps) (256 Kbps-10 Mbps) & Latency (ms) \\
\hline 1 & Microsoft Azure & MS & https://www.azure.microsoft.com & 28.15 & 46.5 \\
\hline 2 & GMOCloud -US & GMO & https://www.us.gmocloud.com & 1.31 & 506 \\
\hline 3 & HP Cloud & HP & https://www.hpcloud.com & 22.64 & 16 \\
\hline 4 & Amazone S3 & Amz & https://www.aws.amazon.com/s3/ & 36.3 & 40.5 \\
\hline 5 & Rackspace Cloud & Rsp & https://www.rackspace.com & 3.07 & 630 \\
\hline 6 & Google Cloud Storage & Ggl & https://www.cloud.google.com & 270.05 & 35 \\
\hline 7 & City Cloud & Cit & https://www.citycloud.com & 8.62 & 89 \\
\hline 8 & Cloud Sigma & Sig & https://www.cloudsigma.com & 24.13 & 215 \\
\hline 9 & Elastic Host & Ela & https://www.elastichosts.com & 12.53 & 34 \\
\hline 10 & CenturylinkCloud & Cent & https://www.centurylinkcloud.com & 254.79 & 36 \\
\hline 11 & Digital Ocean & Dig & https://www.digitalocean.com & 4.22 & 190 \\
\hline
\end{tabular}

TABLE III

Service MeAsurement By CloudAuditor 1

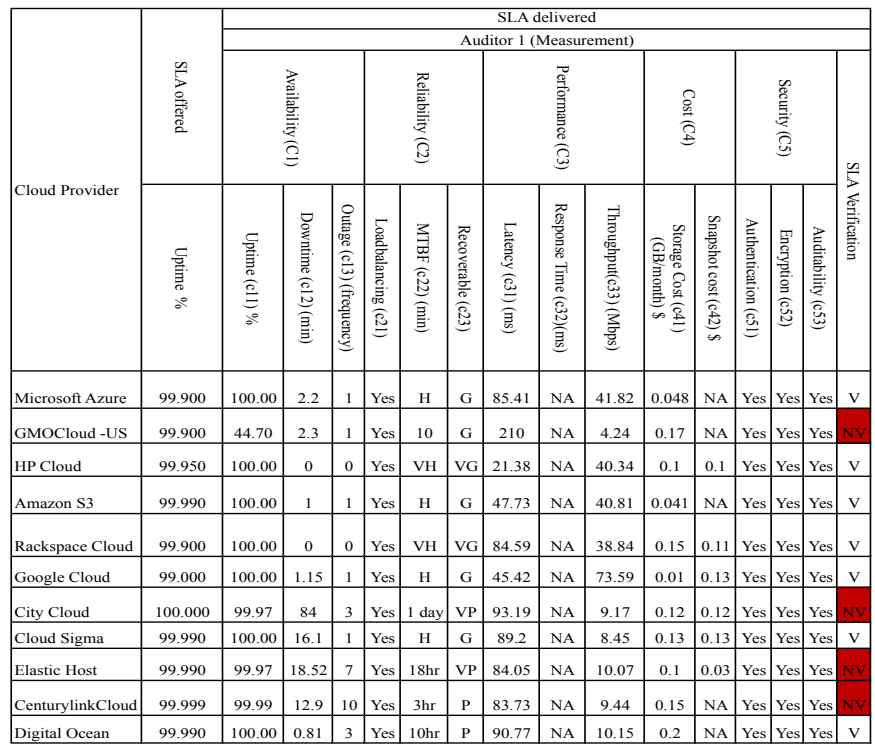

provider are different for each cloud auditor because of different monitoring environment. In some cases the results are conflicting. The cost are directly referred from their websites. Service measurement from all selected cloud providers covers only the computing and storage services. Ranking order is based on the data gathered over a period of seven days. The actual ranking could change when considering a another or longer observation period or using input from other auditors. As a result, the presented results is only for explanatory purposes and should not be considered in any case as conclusive regarding the real QoS of the service providers.

Besides the evaluations of auditors, service experience by cloud users may be similarly included in the overall evaluation of cloud providers by the way of user surveys. This part is yet to be completed in our ongoing research work [24].

\section{B. Service Verification}

For the service verification, the measured value for criteria $C_{1}$ (see Table III and Table IV) is used, as all the selected cloud providers offer monthly uptime in SLA com-
TABLE IV

Service Measurement By CloudAuditor 2

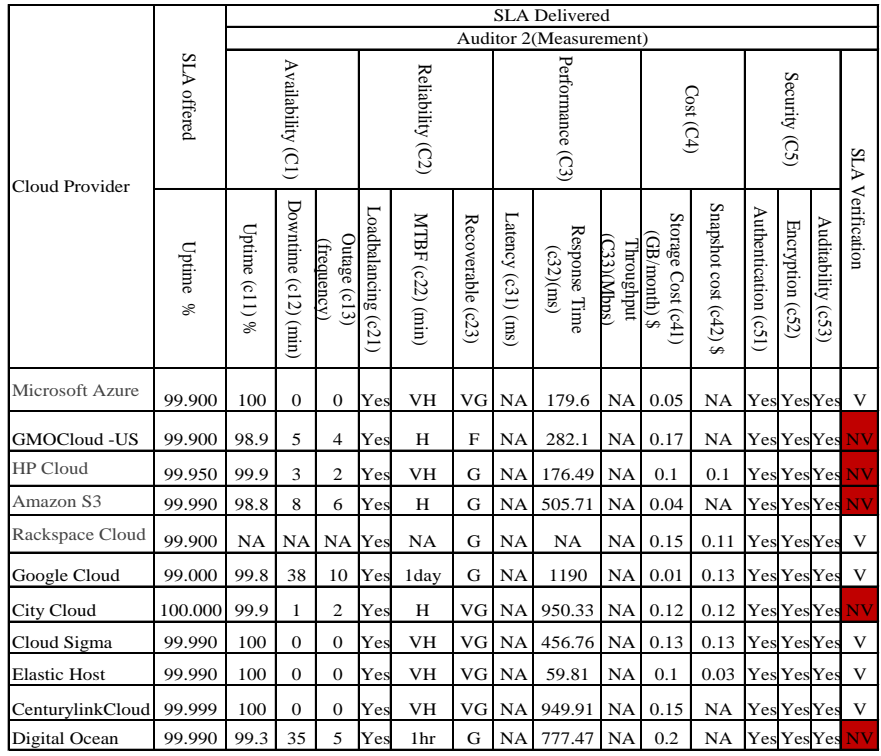

mitment. Measured value for criteria $C_{1}$ is mapped to criterion Availability and compared with SLA offered by cloud providers. A value with red background shows the noncompliance of an SLA and is a subject to penalty for SLA violation. ' $V$ ' represents a positive verification of a service and ' $N V$ ' represents the negative verification of services. In data observed by CloudAuditor 1 ; GMOCloud-US, City Cloud, Elastic Host and Centurylink Cloud did not comply the service commitment as stated in their SLA. There is a significant gap between service offer and service delivered in GMOCloud-US and a comparatively less sever violation in the cases of City Cloud and Elastic Host. The difference is little for Centurylink Cloud, but it still did not manage to comply with its SLA. Similarly, GMOCloud-US, HP Cloud, Amazon S3 City Cloud and Digital Ocean did not comply the service commitment in service observation by CloudAuditor ${ }_{2}$. There is a significant difference in offered SLA and delivered availability in GMOCloud-US, Amazon S3 and Digital Ocean but it is less significant in HP Cloud and City Cloud. Unless cloud providers commit to all selected quality criteria services in 
TABLE V

SERVICE MAPPING TO ORDINAL VALUE MEASUREMENT By CloudAuditor 1

\begin{tabular}{|c|c|c|c|c|c|c|c|c|c|c|c|c|c|c|c|c|}
\hline \multirow{4}{*}{ Cloud Provider } & \multirow{4}{*}{ 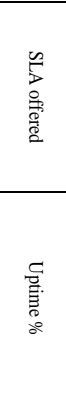 } & \multicolumn{15}{|c|}{ SLA delivered } \\
\hline & & \multicolumn{15}{|c|}{ Auditor 1 (Mapped) } \\
\hline & & \multicolumn{3}{|c|}{ 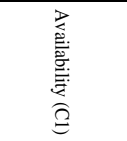 } & \multicolumn{3}{|c|}{ 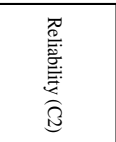 } & \multicolumn{3}{|c|}{ 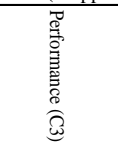 } & \multicolumn{2}{|c|}{$\begin{array}{l}\stackrel{2}{ڤ} \\
\stackrel{\leftrightarrow}{\rightleftarrows}\end{array}$} & \multicolumn{3}{|c|}{ 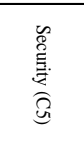 } & \multirow{2}{*}{ 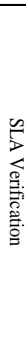 } \\
\hline & & 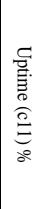 & 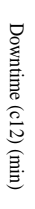 & 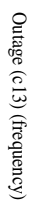 & 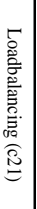 & 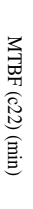 & 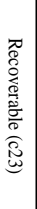 & 产 & 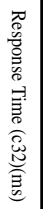 & 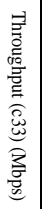 & 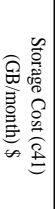 & 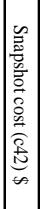 & 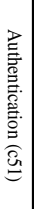 & 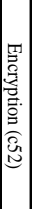 & 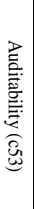 & \\
\hline Microsoft Azure & 99.900 & 4.00 & 3 & 3 & 4 & 3 & 3 & 3 & NA & 4 & 4 & $\mathrm{NA}$ & 4 & 4 & 4 & $\mathrm{v}$ \\
\hline GMOCloud -US & 99.900 & 0.00 & 3 & 3 & 4 & 1 & 3 & 2 & NA & 1 & 3 & $\mathrm{NA}$ & 4 & 4 & 4 & NI \\
\hline HP Cloud & 99.950 & 4.00 & 4 & 4 & 4 & 4 & 4 & 4 & NA & 4 & 3 & 4 & 4 & 4 & 4 & $\mathrm{~V}$ \\
\hline Amazon S3 & 99.990 & 4.00 & 3 & 3 & 4 & 3 & 3 & 4 & NA & 4 & 4 & $\mathrm{NA}$ & 4 & 4 & 4 & $\mathrm{~V}$ \\
\hline Rackspace Cloud & 99.900 & 4.00 & 4 & 4 & 4 & 4 & 4 & 3 & NA & 4 & 3 & 4 & 4 & 4 & 4 & $\mathrm{~V}$ \\
\hline Google Cloud & 99.000 & 4.00 & 3 & 3 & 4 & 3 & 3 & 4 & $\mathrm{NA}$ & 4 & 4 & 4 & 4 & 4 & 4 & $\mathrm{~V}$ \\
\hline City Cloud & 100.00 & 1.00 & 0 & 2 & 4 & 2 & 1 & 3 & NA & 2 & 3 & 4 & 4 & 4 & 4 & NV \\
\hline Cloud Sigma & 99.990 & 4.00 & 2 & 3 & 4 & 3 & 3 & 3 & NA & 2 & 3 & 4 & 4 & 4 & 4 & $\mathrm{~V}$ \\
\hline Elastic Host & 99.990 & 2.00 & 2 & 1 & 4 & 3 & 1 & 3 & NA & 2 & 3 & 4 & 4 & 4 & 4 & \\
\hline CenturylinkCloud & 99.999 & 2.00 & 1 & 0 & 4 & 2 & 2 & 3 & NA & 2 & 3 & $\mathrm{NA}$ & 4 & 4 & 4 & IV \\
\hline Digital Ocean & 99.990 & 4.00 & 0 & 2 & 4 & 3 & 2 & 3 & NA & 2 & 3 & $\mathrm{NA}$ & 4 & 4 & 4 & $\mathrm{~V}$ \\
\hline
\end{tabular}

TABLE VI

SERVICE MAPPING TO ORDINAL VALUE MEASUREMENT BY CloudAuditor $_{2}$

\begin{tabular}{|c|c|c|c|c|c|c|c|c|c|c|c|c|c|c|c|c|}
\hline \multirow[b]{4}{*}{ loud Provider } & \multirow{4}{*}{ 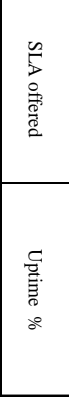 } & \multirow{2}{*}{\multicolumn{15}{|c|}{$\begin{array}{l}\text { SLA Delivered } \\
\text { Auditor 2(Mapped)) }\end{array}$}} \\
\hline & & & & & & & & & & & & & & & & \\
\hline & & \multicolumn{3}{|c|}{ 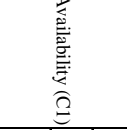 } & \multicolumn{3}{|c|}{ 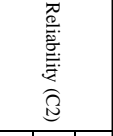 } & \multicolumn{3}{|c|}{ 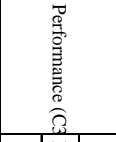 } & \multicolumn{2}{|c|}{$\frac{\delta}{\stackrel{\delta}{\hbar}}$} & \multicolumn{3}{|c|}{ 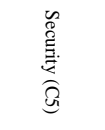 } & \multirow[b]{2}{*}{-} \\
\hline & & 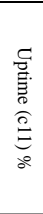 & 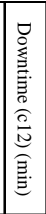 & 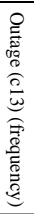 & 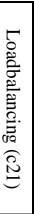 & 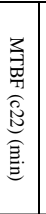 & 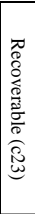 & 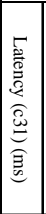 & 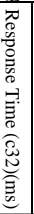 & 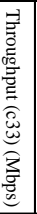 & 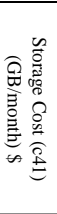 & 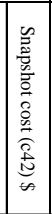 & 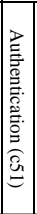 & 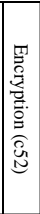 & 离 & \\
\hline Microsoft Azure & 99.900 & 4 & 4 & 4 & 4 & 4 & 4 & $\mathrm{NA}$ & 4 & $\mathrm{NA}$ & 4 & $\mathrm{NA}$ & 4 & 4 & 4 & V \\
\hline GMOCloud -US & 99.900 & 1 & 3 & 3 & 4 & 3 & 2 & $\mathrm{NA}$ & 4 & $\mathrm{NA}$ & 3 & $\mathrm{NA}$ & 4 & 4 & 4 & \\
\hline HP Cloud & 99.950 & 3 & 3 & 3 & 4 & 4 & 3 & $\mathrm{NA}$ & 4 & $\mathrm{NA}$ & 3 & 4 & 4 & 4 & 4 & \\
\hline Amazon S3 & 99.990 & 2 & 2 & 2 & 4 & 3 & 3 & $\mathrm{NA}$ & 3 & $\mathrm{NA}$ & 4 & $\mathrm{NA}$ & 4 & 4 & 4 & \\
\hline Rackspace Cloud & 99.900 & NA & NA & $\mathrm{NA}$ & 4 & $\mathrm{NA}$ & 3 & $\mathrm{NA}$ & $\mathrm{NA}$ & $\mathrm{NA}$ & 3 & 4 & 4 & 4 & 4 & $\mathrm{v}$ \\
\hline Google Cloud & 99.000 & \begin{tabular}{|l|}
4 \\
\end{tabular} & 1 & 1 & 4 & 2 & 3 & $\mathrm{NA}$ & 2 & $\mathrm{NA}$ & 4 & 4 & 4 & \begin{tabular}{|l|}
4 \\
\end{tabular} & 4 & $\mathrm{v}$ \\
\hline City Cloud & 100.00 & 3 & 3 & 3 & 4 & 3 & 4 & $\mathrm{NA}$ & 2 & $\mathrm{NA}$ & 3 & 4 & 4 & 4 & 4 & \\
\hline \begin{tabular}{|l|} 
Cloud Sigma \\
\end{tabular} & 99.990 & 4 & 4 & 4 & 4 & 4 & 4 & $\mathrm{NA}$ & 3 & $\mathrm{NA}$ & 3 & 4 & 4 & 4 & 4 & $\mathrm{~V}$ \\
\hline Elastic Host & 99.990 & 4 & 4 & 4 & 4 & 4 & 4 & $\mathrm{NA}$ & 4 & $\mathrm{NA}$ & 3 & 4 & 4 & 4 & 4 & V \\
\hline enturylinkCloud & 99.999 & 4 & 4 & 4 & 4 & 4 & 4 & $\mathrm{NA}$ & 2 & $\mathrm{NA}$ & 3 & $\mathrm{NA}$ & 4 & 4 & 4 & \\
\hline & & & & & & & & & & & & & & & & \\
\hline
\end{tabular}

their SLA, which is not the case in practice, it is not possible to provide the verification of service delivery for all SLA parameters. Instead of that, we propose an evaluation model to provide the status of commercial available cloud providers as a performance heat map. The visual performance heat map is used to recommend the cloud services to the cloud brokers and cloud users.

\section{Service Quality Evaluation}

Table VII shows mapping of measured values into an ordinal performance scale consisting of five levels: from Very Poor (0) to Very Good (4). According to service commitment by cloud providers, multiple contract breaking points are defined and the granted service credit are offered according to the type of service violation. For example Amazon-S3 (www.aws.amazon.com/s3/sla) guarantee $99.95 \%$ monthly uptime and it provides the service credit according to uptime $\%$. If uptime \% is less than 99.95 but equal to or greater than 99.0 , the service credit is $10 \%$. If uptime $\%$ is less than 99.0 the service credit is $30 \%$. To consider all the values defined in SLA offer, minimum value ( min $\left._{\text {value }}\right)$, maximum value $\left(\max _{\text {value }}\right)$ and threshold value $\left(t h_{\text {value }}\right)$ are set to comparing SLAs of cloud providers. If a measured value is greater than max $_{\text {value }}$ it is interpreted as Very Good (VG). If measured value is less than min value it is interpreted as very poor (VP). If measurements of parameters are missing for cloud provider, it is mapped to No Value ' $N A^{\prime}$. The third column in Table VII gives an interpretation of measured SLA parameter after comparing with offered SLA to ordinal values. The service mapping to ordinal value by CloudAudior ${ }_{1}$ and CloudAudior $_{2}$ are presented in Table V and VI respectively.

As monitoring systems of CloudAuditor ${ }_{1}$ and CloudAudiotor $_{2}$ are different, the mapping from measurement from Table III to Table $\mathrm{V}$ and from Table IV to Table VI are independent. Max value, min $_{\text {value }}$ and $t h_{\text {value }}$ values for each sub-criteria are defined for each cloud auditor. According to performance recorded in Table III and IV, ordinal values are mapped to Table V and VI, respectively. For example, in Table V, Uptime value of Microsoft Azure measured by CloudAudior $_{1}$ is $>\max _{\text {value }}$, so it is mapped to ordinal level '4'. Downtime, Outage Frequency, MTBF, Recoverable, Latency are in the range of $\max _{\text {value }}$ and mapped to ordinal level ' 3 '. Costs of the delivered services, gathering cost of storage and of VM instance, are taken from the providers' websites. Mapping of Downtime, Outage Frequency, Latency, Response Time and Cost is reverse to other sub-criteria mapping because their values should be minimized. The performance on the Security criterion considers the sub-criteria: Authentication, Encryption, and Auditability. Authentication protocol, encryption algorithm as well as logs provided during service usage are considered in order to evaluate the performance on these sub-criteria; two options: ' $Y E S$ ' or ' $N O$ ' are chosen as the evaluation scale. During our actual observation period, all of the eleven cloud providers were found satisfactory in terms of Security.

\section{CSP SELECTION AIDING APPROACH}

The primary goal of this work is to help cloud users and cloud brokers with selection of the best cloud providers based on the service offer, delivery and user experience according to the defined SLA criteria. We follow a decision aiding approach proposed by Bisdorff [25], which involves the following steps:

1) Sorting the potential cloud providers into marginal performance quantiles classes; 
TABLE VII

ORDINAL LEVEL AND INTERPRETATION OF AUDITOR MEASUREMENT IN ORDinAL VALUE

\begin{tabular}{|l|l|l|}
\hline Linguistic terms & Ordinal Value & Measured Value \\
\hline Very poor(VP) & 0 & $<$ min $_{\text {value }}$ \\
Poor (P) & 1 & min $_{\text {value }}$ \\
Fair (F) & 2 & th $_{\text {value }}$ \\
Good(G) & 3 & max $_{\text {value }}$ \\
Very Good(VG) & 4 & $>$ max $_{\text {value }}$ \\
No Value & NA & Data not received \\
\hline
\end{tabular}

\begin{tabular}{|c|c|c|c|c|c|c|c|c|c|c|c|c|c|c|}
\hline criteria & tpt & upT & MTBF & Lat & Rev & ouT & dwT & stC & auD & enC & auT & snC & rsT & LB \\
\hline weights & 2.00 & 2.00 & 2.00 & 2.00 & 2.00 & 2.00 & 2.00 & 3.00 & 2.00 & 2.00 & 2.00 & 3.00 & 2.00 & 2.00 \\
\hline \hline tau $\left.^{*}\right)$ & 0.62 & 0.59 & 0.54 & 0.45 & 0.43 & 0.43 & 0.43 & 0.36 & 0.00 & 0.00 & 0.00 & 0.00 & 0.00 & 0.00 \\
\hline \hline Amz & 4 & 4 & 3 & 4 & 3 & 3 & 3 & 4 & 4 & 4 & 4 & NA & NA & 4 \\
\hline \hline MS & 4 & 4 & 3 & 3 & 3 & 3 & 3 & 4 & 4 & 4 & 4 & NA & NA & 4 \\
\hline \hline HP & 4 & 4 & 4 & 4 & 4 & 4 & 4 & 3 & 4 & 4 & 4 & 4 & NA & 4 \\
\hline \hline Rsp & 4 & 4 & 4 & 3 & 4 & 4 & 4 & 3 & 4 & 4 & 4 & 4 & NA & 4 \\
\hline \hline Ggl & 4 & 4 & 3 & 4 & 3 & 3 & 3 & 4 & 4 & 4 & 4 & 4 & NA & 4 \\
\hline \hline Sig & 2 & 4 & 3 & 3 & 3 & 3 & 2 & 3 & 4 & 4 & 4 & 4 & NA & 4 \\
\hline \hline Dig & 2 & 4 & 3 & 3 & 2 & 2 & 0 & 3 & 4 & 4 & 4 & NA & NA & 4 \\
\hline \hline Ela & 2 & 2 & 3 & 3 & 1 & 1 & 2 & 3 & 4 & 4 & 4 & 4 & NA & 4 \\
\hline \hline Cent & 2 & 2 & 2 & 3 & 2 & 0 & 1 & 3 & 4 & 4 & 4 & NA & NA & 4 \\
\hline \hline Cit & 2 & 1 & 2 & 3 & 1 & 2 & 0 & 3 & 4 & 4 & 4 & 4 & NA & 4 \\
\hline \hline GMO & 1 & 0 & 1 & 2 & 3 & 3 & 3 & 3 & 4 & 4 & 4 & NA & NA & 4 \\
\hline Color legend:
\end{tabular}

Fig. 3. Heat map table by Auditor 1

2) Ranking the providers with multiple ordinal performance criteria;

3) Sorting the performance criteria in decreasing order of correlation with the previous ranking;

4) Visualizing the results in a performance heat map, ordering the potential CSPs from the best to the worst alternative.

\section{A. Sorting marginal performances into quantile classes}

Let $X$ be the set of $n$ potential cloud providers evaluated on a single real performance criteria. We denote $x, y, \ldots$ the performances observed of the potential decision actions in $X$. We call quantile $q(p)$ the performance such that $p \%$ of the observed $n$ performances in $X$ are less or equal to $q(p)$. The quantile $q(p)$ is estimated by linear interpolation from the cumulative distribution of the performances in $X$.

Consider a series: $p_{k}=k / q$ for $k=0, \ldots q$ of $q+1$ equally spaced quantiles, like quartiles: $0,0.25,0.5,0.75,1.0$; quintiles: $0,0.2,0.4,0.6,0.8,1.0$; or deciles: $0,0.1,0.2, \ldots$, $0.9,1.0$. The upper closed ${ }^{1} q^{k}$ class corresponds to the interval ]$\left.q\left(p_{k-1}\right) ; q\left(p_{k}\right)\right]$, for $k=2, \ldots, q$, where $\left.q\left(p_{q}\right)=\max _{X} x\right)$ and the first class gathers all data below $\left.\left.p_{1}:\right]-\infty ; q\left(p_{1}\right)\right]$. We call $q$-tiles a complete series of $k=1, \ldots, q q_{k}$ quantile classes. For the performance heat map visualization, we associate to each of such $p^{k}$ quantile class a color from dark red (worst) to dark green (best). See for instance the color legend for 7-tiles in Fig. 3, 4 and 5.

\footnotetext{
${ }^{1}$ The lower closed $q_{k}$ class corresponds to the interval $\left[q\left(p_{k-1}\right) ; q\left(p_{k}\right)[\right.$.
}

On each criteria we thus associate to the performance $x$ of a cloud provider the color of the qtiles class to which belongs $x$. In Fig 3 again, we may thus observe that, for CloudAuditor $_{1}$, providers $A m z, M S, H P, R s p$ and $G g l$ show on sub-criteria tpT:Performance, Amz, MS, HP, Rsp, Ggl, Sig and Dig show on sub-criteria upT:Availability, $H P$ and Rsp show on sub-criteria $M T B F$ :Reliability, $A m z, H P$ and $G g l$ show on sub-criteria Lat:Performance the best performance (4), whereas cloud provider $G M O$ shows the worst performance in all the cases but cloud provider GMO shows worst performance only on sub-criteria Rcv:Reliability observed by Auditor $_{2}$ (see Fig 4). Similarly, in Fig. 5 we observed that, for combining result of both auditors, providers $M S, A M Z$, $H P, G g l$ and $R s p$ show on sub-criteria tpT:Performance, $H P$ and $R s p$ show on sub-criteria MTBF:Reliability, Amz, $H P$ and $G g l$ show on sub-criteria Lat:Performance, $M S$, $A m z, H P, G g l, R s p$, Sig and Gig show on sub-criteria upT:Availability and MS, Cent, Sig, Ela and Cit show on sub-criteria Rcv:Reliability the best performance (4), whereas provider GMO shows the worst performance in all the cases.

It is worthwhile mentioning that the quantiles sorting result has not to be taken as a kind of service rating. When observing in the heat map table that a CSP is evaluated best on a criteria, this only means that its performance is to be considered best relatively to the actually given set of potentially available CSPs. That is why, in case of identical evaluation of all potential CSPs, the performance is sorted into the best quantile for all of them (see last rows in Fig. 3, 4 and 5).

\section{B. Multiple Criteria Ranking the CSPs}

In Fig. 3 and 4, the CSPs appear ranked in decreasing order from the overall best to overall worst performing. This overall ranking is computed from bipolar outranking situations [26], where we consider that an alternative $x$ outranks an alternative $y$ when there is a significant majority of criteria that warrant a 'better than' relation between them and there is no considerable counter-performance observed between when considering $x$ in place of $y$.

When computing these outranking relation, we consider the five main performance criteria, namely Availability, Reliability, Performance, Cost and Security to be equally important; and all sub-criteria within each criterion are considered to be equisignificant. All criteria has the three sub-criteria except $\operatorname{Cost}\left(C_{4}\right)$. Weights are assigned to each sub-criteria to make equally significant for all criteria (see second row in Fig. 3, 4 and 5). Thus we obtain the following set of significance weights of the criteria and sub-criteria: $w_{A}=w_{c 11}+w_{c 12}+$ $w_{c 13}=w_{R}=w_{c 21}+w_{c 22}+w_{c 23}=w_{P}=w_{c 31}+w_{c 32}+$ $w_{c 33}=w_{C}=w_{c 41}+w_{c 42}=w_{S}=w_{c 51}+w_{c 52}+w_{c 53}$ where $w c_{i j}$ represents the significance weight assigned to subcriterion $j$ under criterion $i$.

Considering eleven potential commercial cloud providers, alternative $A M Z$ is ranked as the highest ranked cloud provider by CloudAuditor ${ }_{1}$, whereas $M S$ is ranked as the highest ranked cloud provider by CloudAuditor 2 (see Fig. 3 and 4), whereas the alternative $G M O$, respectively 


\begin{tabular}{|c|c|c|c|c|c|c|c|c|c|c|c|c|c|c|}
\hline criteria & ouT & dwT & MTBF & upT & Rev & rsT & auD & enC & auT & $\mathbf{s n C}$ & tpT & Lat & LB & stC \\
\hline weights & 2.00 & 2.00 & 2.00 & 2.00 & 2.00 & 2.00 & 2.00 & 2.00 & 2.00 & 3.00 & 2.00 & 2.00 & 2.00 & 3.00 \\
\hline $\operatorname{tau}^{(*)}$ & 0.75 & 0.70 & 0.60 & 0.55 & 0.53 & 0.42 & 0.00 & 0.00 & 0.00 & 0.00 & 0.00 & 0.00 & 0.00 & -0.07 \\
\hline MS & 4 & 4 & 4 & 4 & \begin{tabular}{|l|}
4 \\
\end{tabular} & 4 & 4 & 4 & 4 & NA & NA & \begin{tabular}{|l|} 
NA \\
\end{tabular} & 4 & 4 \\
\hline Cent & 4 & 4 & 4 & 4 & 4 & 4 & 4 & 4 & 4 & NA & NA & NA & 4 & 3 \\
\hline Ela & 4 & 4 & 4 & 4 & 4 & 4 & 4 & 4 & 4 & 4 & NA & NA & 4 & 3 \\
\hline Sig & 4 & 4 & 4 & 4 & 4 & 3 & 4 & 4 & 4 & 4 & NA & NA & 4 & 3 \\
\hline Cit & 3 & 4 & 3 & 3 & 4 & 2 & 4 & 4 & 4 & 4 & NA & NA & 4 & 3 \\
\hline HP & 3 & 3 & 4 & 3 & 3 & 4 & 4 & 4 & 4 & 4 & NA & NA & 4 & 3 \\
\hline Rsp & NA & \begin{tabular}{|l|}
$N A$ \\
\end{tabular} & NA & NA & 3 & NA & 4 & 4 & 4 & 4 & NA & NA & 4 & 3 \\
\hline GMO & \begin{tabular}{|l|}
3 \\
\end{tabular} & 3 & 3 & 1 & 2 & 4 & 4 & 4 & 4 & NA & NA & NA & $\overline{4}$ & 3 \\
\hline Ggl & 1 & 1 & 2 & 4 & 3 & 2 & 4 & 4 & 4 & 4 & NA & NA & 4 & 4 \\
\hline $\mathbf{A m z}$ & 2 & 2 & 3 & 2 & 3 & 3 & 4 & 4 & 4 & NA & NA & NA & 4 & 4 \\
\hline Dig & 1 & 1 & 2 & 2 & 3 & 2 & 4 & 4 & 4 & NA & $\mathrm{NA}$ & NA & 4 & 3 \\
\hline \multicolumn{15}{|c|}{ Color legend: } \\
\hline quantile & 0.12 & & $.29 \%$ & $.43 \%$ & 0.57 & \begin{tabular}{l|l}
$7 \%$ & 0 \\
\end{tabular} & $.71 \%$ & $0.8 c$ & & $1.00 \%$ & & & & \\
\hline
\end{tabular}

(*) tau: Ordinal (Kendall) correlation of marginal criterion and global outranking relation.

Fig. 4. Heat map table by Auditor 2

Dig, is ranked lowest by CloudAuditor ${ }_{1}$, respectively CloudAuditor $_{2}$. Equally spaced 7 quantiles are considered in these heat maps, where, as mentioned before, dark green color indicates the relatively highest performance, whereas dark red indicates the relatively lowest performance.

Collective ranking of the potential eleven cloud providers is shown in Fig. 5 by grouping the evaluations of the two cloud auditors. In the collective ranking, evidently alternative $M S$ is the highest ranked cloud provider, whereas cloud provider alternative $G M O$ is the lowest ranked.

\section{Building a Service Selection RECOMMENDATION}

In the previous section, we present an evaluation model of the delivered cloud service from eleven commercially available cloud providers (see Table II) which is based on their SLAs and service delivery. All the CSPs considered in this paper provide only monthly uptime in percents as the SLA compliance criterion. So, services Availability is taken into account for the verification and compared with the offered SLA commitments. In seven days observation many of the observed cloud providers did not comply their service Availability commitment. CloudAuditor ${ }_{1}$ found four cloud providers who did not comply with their service commitment; whereas CloudAuditor 2 found five cloud providers who did not comply their service commitment. However, the results could change with a longer observation period.

Our selection recommendation consists of an ordered Performance Heat Map (see Fig. 5), which lists the potential set of CSPs sorted in decreasing order of overall evaluation. Remind that dark green, respectively dark red marked performances indicate best ones, respectively worst ones on each sub-criterion. Notice also (see ordinal correlation in row tau in Fig. 5) that Performance subscription Throughput (tpT), Reliability subscription MTBF and performance subscription Latency (Lat) evaluated by CloudAuditor ${ }_{1}$, Storage Costs (stC) and Availability subscription Uptime (upT) evaluated by both auditors, do influence most the recommended consensual ranking. Similarly, sub-criterion Throughput (tpT), Uptime
(upT), MTBF and Latency (Lat) by CloudAuditor ${ }_{1}$ (see Fig. 3) whereas ouT, $d w T, M T B F$ and $u p T$ (see Fig. 4) in performance ranking, play dominance role in sorting order. $H P$ is lower than $M S$ in Fig. 3 because sub-criterion $s t C$ played dominant role in such case. Ranking order of Cent is upper than Ela in Fig. 4 because optimistic sorting approach is applied which makes assumption that many attributes with good performance results good performance in missing parameters. From the view of optimistic sorting approach, Cent and Ela have the same position.

The results of validation process and the heat maps shows the necessity to diversify the input for the recommendation system. The two used cloud auditors presents great discrepancy of results. The advantage of the heat map method is that it can aggregate results of multiple auditors, to present non-trivial change of results. Cloud users and brokers may select the most appropriate cloud services according to their requirements with tradeoff among different requirements; they can base on the ranking of all SLA attributes or base on individual service requirements. Despite that all these service verification and performance rankings are performed in the mentioned circumstances of data observation period, our performance heat maps show a convincing, consensually ranked multiple criteria performance of commercially available CSPs. This visual recommender tool may thus give important hints to cloud users and cloud brokers.

\section{CONCLUSION AND FUtURE WORKS}

The proposed evaluation model is a recommendation system that facilitates selection of the most appropriate cloud service provider to cloud users and cloud brokers. The following important aspects are addressed in cloud provider selection:

The proposed model considers both dynamic and static attributes during the selection of the cloud provider.

It verifies the cloud services delivered by cloud providers according to service details provided in the SLA commitment using multiple cloud auditors.

It proposes an individual heat map performance table and combined heat map performance table which provide the ranking of cloud providers and performance rank of each cloud provider in all the selected SLA attributes.

The performance table provides the recommended cloud providers for the selected SLA attributes and nonrecommended cloud providers for those the SLA attributes. The recommendation can be provided to cloud users and cloud brokers to select cloud services according to their requirements or according to overall performance table.

In this paper, ranking and recommendation is based on the measurements of the service delivery. For the better performance of proposed framework, feedback of cloud users will be included in the future using cloud user survey [24]. This work is a part of work of the SLA-assured brokering and CSP certification framework [27]. SLA-assured broker uses the service recommendation table to propose the cloud services according to the cloud users requirements. Penalty calculation according to service violation by cloud providers is also a 


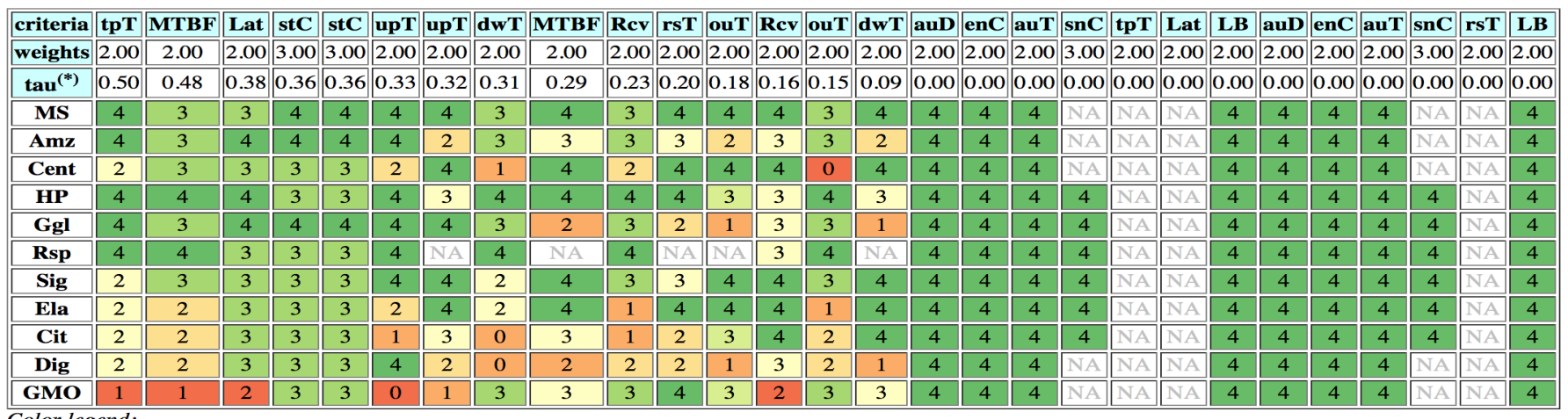

\begin{tabular}{|l|c|}
\hline GMO & 1 \\
\hline Color legend:
\end{tabular}

\begin{tabular}{|l|l|l|l|l|l|l|l|}
\hline quantile & $0.14 \%$ & $0.29 \%$ & $0.43 \%$ & $0.57 \%$ & $0.71 \%$ & $0.86 \%$ & $1.00 \%$ \\
\hline
\end{tabular}

(*) tau: Ordinal (Kendall) correlation of marginal criterion and global outranking relation.

Fig. 5. Heat map table by All Auditors

potential future work, together with multi-criteria optimization of cloud brokering problems [4].

\section{ACKNOWLEDGMENT}

This work was partially funded from Luxembourg National Research Fund (FNR) IShOP (POLLUX/13/IS/6466384) project. S. S. Wagle would like to thank LAST-JD program for financially supporting to perform this research.

\section{REFERENCES}

[1] "Qos modeling for green scheduling in clouds," 2015 "http://homepages.laas.fr/tguerout/data/PUBLIS/REVUES/SUSCOM EARMS QoS Modeling for Green Scheduling in Clouds.pdf".

[2] C. Kotsokalis, J. Rueda, S. Gmez, and A. Chimeno, "Penalty management in the sla@soi project," in Service Level Agreements for Cloud Computing, 2011.

[3] M. Guzek, A. Gniewek, P. Bouvry, J. Musial, and J. Blazewicz, "Cloud brokering: Current practices and upcoming challenges," IEEE Cloud Computing, vol. 2, no. 2, pp. 40-47, Mar 2015.

[4] M. Guzek, P. Bouvry, and E.-G. Talbi, "A survey of evolutionary computation for resource management of processing in cloud computing [review article]," IEEE Computational Intelligence Magazine, vol. 10, no. 2, pp. 53-67, May 2015.

[5] M. Guzek, J. E. Pecero, B. Dorronsoro, and P. Bouvry, "Multi-objective evolutionary algorithms for energy-aware scheduling on distributed computing systems," Applied Soft Computing, vol. 24, pp. 432-446, 2014.

[6] P. Wang, "Qos-aware web services selection with intuitionistic fuzzy set under consumers vague perception," Expert Systems with Applications, vol. 36, no. 3, Part 1, pp. 4460 - 4466, 2009.

[7] S. Garg, S. Versteeg, and R. Buyya, "Smicloud: A framework for comparing and ranking cloud services," in Fourth IEEE International Conference on Utility and Cloud Computing (UCC), 2011, pp. 210218.

[8] "C. S. M. I. C. (csmic), smi framework," 2014, "http://betawww.cloudcommons.com/servicemeasure mentindex".

[9] S. Thomas, "Theory and applications of analytic network process." Pittsburgh, PA: RWS Publications, 2005.

[10] A. Li, X. Yang, S. Kandula, and M. Zhang, "Cloudcmp: Comparing public cloud providers," in Proceedings of the 10th ACM SIGCOMM Conference on Internet Measurement, ser. IMC, 2010, pp. 1-14.

[11] M. Guzek, S. Varrette, V. Plugaru, J. E. Pecero, and P. Bouvry, "A holistic model of the performance and the energy efficiency of hypervisors in a high-performance computing environment," Concurrency and Computation: Practice and Experience, vol. 26, no. 15, pp. 2569-2590, 2014.
[12] H. Chan and T. Chieu, "Ranking and mapping of applications to cloud computing services by svd," in IEEE/IFIP Network Operations and Management Symposium Workshops (NOMS Wksps), April 2010, pp. 362-369.

[13] P. Choudhury, M. Sharma, K. Vikas, T. Pranshu, and V. Satyanarayana, "Service ranking systems for cloud vendors," in Advanced Materials Research, Vols. 433-440, January 2012, pp. 3949-3953.

[14] Z. Zheng, Y. Zhang, and M. Lyu, "Cloudrank: A qos-driven component ranking framework for cloud computing," in 29th IEEE Symposium on Reliable Distributed Systems, Oct 2010, pp. 184-193.

[15] L. Qu, Y. Wang, M. A. Orgun, L. Liu, and A. Bouguettaya, "Cloud service selection based on contextual subjective assessment and objective assessment," in Proceedings of the 2014 International Conference on Autonomous Agents and Multi-agent Systems, ser. AAMAS '14, 2014, pp. $1483-1484$.

[16] "Cloud computing reference architecture," 2011, NIST, "http://www.nist.gov/customcf/get pdf.cfm?pub id=909505".

[17] "Practical guide to cloud service agreements version 2.0," 2015, CSCC, "http://www.cloud-council.org/".

[18] "Cloud computing reference architecture," 2015, TM Forum, "http://www.tmforum.org/".

[19] "Cloud computing service level agreements," 2015, EC, "http://ec.europa.eu".

[20] "OCCI working group," "http://occi-wg.org", year=2015.

[21] J. Padhye, V. Firoiu, D. Towsley, and J. Kurose, "Modeling tcp throughput: A simple model and its empirical validation," SIGCOMM Comput. Commun. Rev., vol. 28, no. 4, pp. 303-314, Oct. 1998.

[22] R. Wilhelm, J. Engblom, A. Ermedahl, N. Holsti, S. Thesing, D. Whalley, G. Bernat, C. Ferdinand, R. Heckmann, T. Mitra, F. Mueller, I. Puaut, P. Puschner, J. Staschulat, and P. Stenström, "The worstcase execution-time problem\&mdash;overview of methods and survey of tools," ACM Trans. Embed. Comput. Syst., vol. 7, no. 3, pp. 36:1-36:53, May 2008.

[23] B. Schroeder and G. Gibson, "A large-scale study of failures in highperformance computing systems," IEEE Transactions on Dependable and Secure Computing, vol. 7, no. 4, pp. 337-350, Oct 2010.

[24] S. S. Wagle, M. Guzek, and P. Bouvry, "Cloud service providers ranking based on service delivery and consumer experience," in IEEE 4th International Conference on Cloud Networking (CloudNet) (CLOUDNET), Niagara Falls, Canada, Oct. 2015, pp. 202-205.

[25] R. Bisdorff, "The euro 2004 best poster award: Choosing the best poster in a scientific conference," in Evaluation and Decision Models with Multiple Criteria, ser. International Handbooks on Information Systems, 2015.

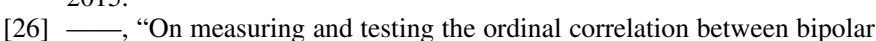
outranking relations," in From Multiple Criteria Decision Aid to Preference Learning, NOV 2012, pp. 91-100.

[27] S. Wagle, "Sla assured brokering (sab) and csp certification in cloud computing," in IEEE/ACM 7th International Conference on Utility and Cloud Computing (UCC), Dec 2014, pp. 1016-1017. 